\title{
On-line Luminosity Measurements at LEP
}

\author{
P. Castro, B. Dehning, G.P. Ferri, P. Puzo \\ CERN, CH-1211 Geneva 23, Switzerland
}

\begin{abstract}
At each LEP interaction point, the luminosity is monitored on-line by small angle Bhabha detectors. These detectors are optimized to observe in all bunches relative luminosity changes in a few seconds. The description of the detectors is given, together with the method used to calculate the luminosity after background correction. Optimization of the LEP performances was done with beam separation scans using the luminosity measurements. Those scans also provide a unique measurement of the vertical beam size at the interaction point.
\end{abstract}

\section{INTRODUCTION}

The aim of the on-line LEP luminosity monitors is to provide precise relative measurements of the luminosity at the four interaction points. These detectors measure the rate of elastic scattering of electron-positron pairs (Bhabha scattering). In order to get high Bhabha scattering rates, the detectors are placed at the very forward regions of the interaction points, very close to the circulating beams. For scattering angles $\theta$ less than $50 \mathrm{mrad}$ the Bhabha scattering is dominated by the Coulomb contribution(1). The differential cross section can be approximated by :

$$
\frac{d \sigma}{d \Omega} \simeq \frac{4 \alpha^{2}}{E^{2} \theta^{4}}
$$

were $E$ is the beam energy and $\alpha$ the fine structure constant.

The setup is shown schematically in Fig. 1. Each luminosity monitor consists of two calorimeters located at opposite sides of the interaction point, one internal and one external to the orbit in the horizontal plane. Two separate monitors are used at each interaction point : monitor 1 detects coincidences of $\mathrm{e}^{+} \mathrm{e}^{-}$pairs with the positron outside and the electron inside as it is shown in Fig. 1, while monitor 2 detects $\mathrm{e}^{+} \mathrm{e}^{-}$pairs with the positron inside and the electron outside. Using both detectors allows to increase the statistical precision on the measurement and decrease systematic effects.

In order to measure the high flux of Bhabha scattered $\mathrm{e}^{+} \mathrm{e}^{-}$pairs at very small scattering angles, the detectors are housed inside horizontal collimators placed inside the vacuum chamber $8.3 \mathrm{~m}$ away from both sides of each interaction point. As part of the horizontal collimators, the detectors can be moved as close as $30 \mathrm{~cm}$ of the circulating beams. The vertically focussing superconducting quadrupole QS0, located between the interaction point and the detectors, decreases the minimum scattered angle accessible on the detector in the horizontal plane. Finally, the detectors intercept Bhabha $\mathrm{e}^{+} \mathrm{e}^{-}$pairs scattered at angles between 2 and $5 \mathrm{mrad}$. 


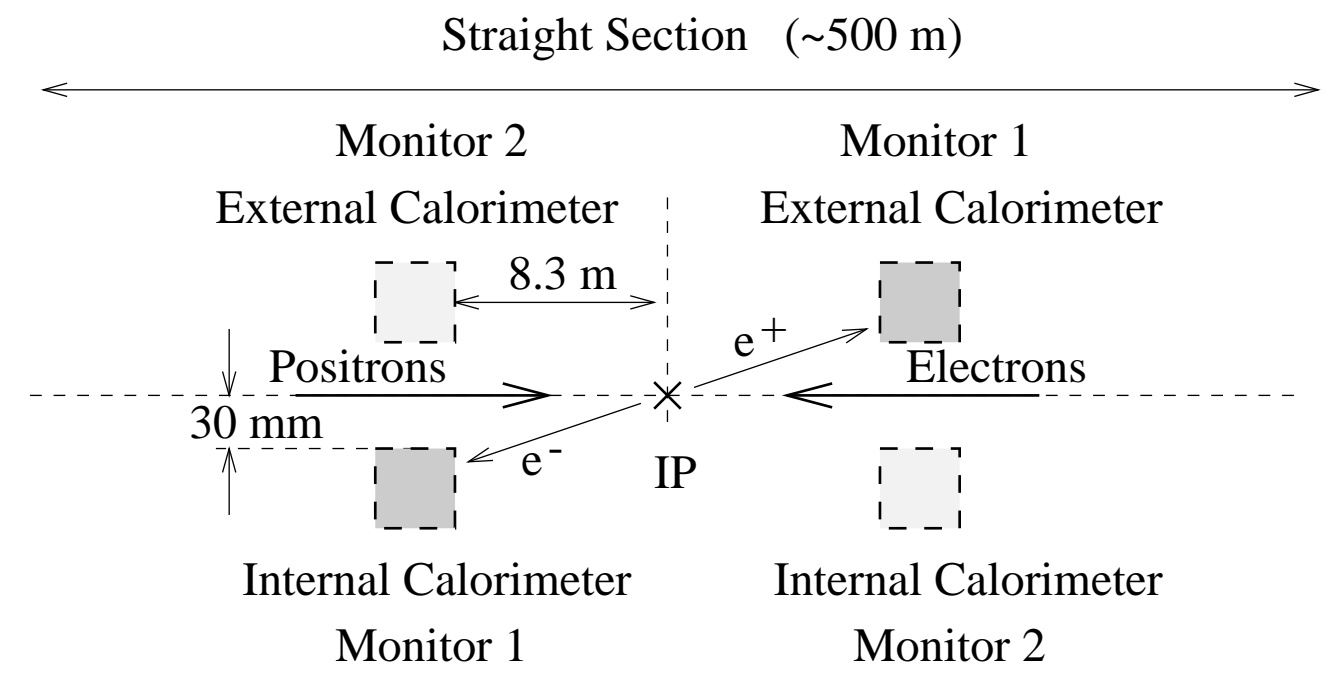

Figure. 1. Top view (not to scale) of four calorimeters installed at each of the four LEP Interaction Points.

The Bhabha rate is typically about $60 \mathrm{~Hz}$ for a luminosity of $10^{31} \mathrm{~cm}^{-2} \mathrm{~s}^{-1}$. Thus, luminosity measurements with $5 \%$ statistical error are obtained in a few seconds. The total cross section of each monitor is about $3.8 \pm 0.2 \mu \mathrm{b}$ in data taking conditions.

In 1995, LEP was mainly running with 12 bunches per beam. Three bunches are grouped together with 250 ns separation (Families) making four groups (Trains) separated by $22 \mu \mathrm{s}$. The luminosity monitors are able to measure luminosity for all 12 bunch crossings per revolution.

\section{GLOBAL SYSTEM}

The system of all LEP luminosity monitors is shown schematically in Fig. 2. In each interaction point, the signals from both pairs of calorimeters are treated separately by a microprocessor in a shielded gallery, $40 \mathrm{~m}$ away from the detectors. This microprocessor performs some first stage calculations and sends data every $9 \mathrm{~s}$ over the network to a main workstation in the control room. The global computations are done there, allowing on line display of the luminosity every $9 \mathrm{~s}$.

\section{DETECTORS}

The detectors used for luminosity monitoring are calorimeters made of tungsten and thin layers of silicon semiconductors. The calorimeters are $86 \mathrm{~mm}$ long and $41 \mathrm{~mm}$ wide to fit in the limited size of the collimators. The description of the detectors and their associated front-end electronics are reported in detail 


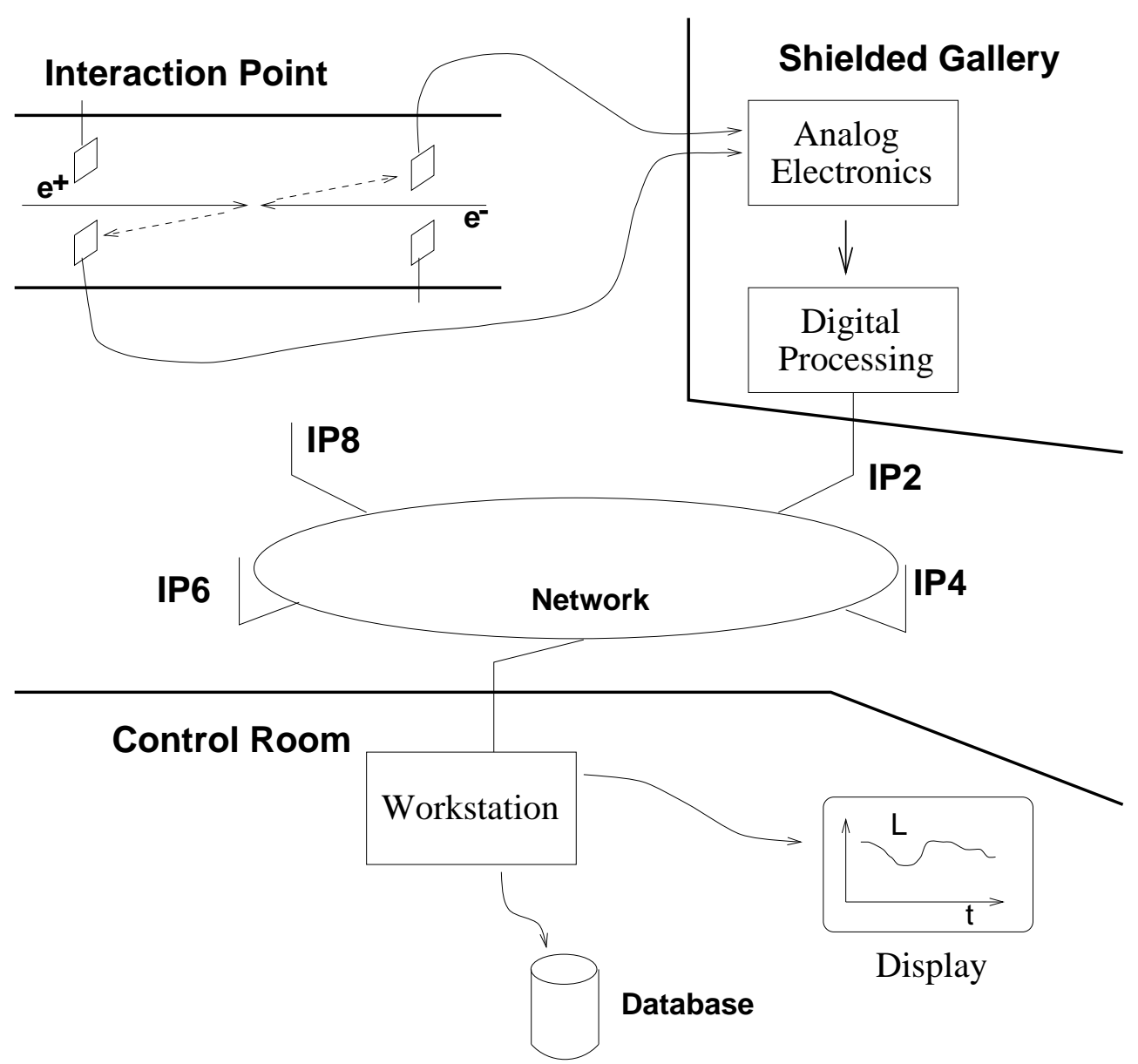

Figure. 2. Schematic view of the measuring system of the LEP luminosity monitors.

in ref.(2).

A silicon detector is mounted after five radiation lengths, close to the location of the maximum number of $\mathrm{e}^{+} \mathrm{e}^{-}$pairs in the longitudinal profile.

When the particles created in the tungsten during the cascade development enter the silicon detector, they create electron-hole pairs that are collected by the reversed bias voltage. One single output channel collects the signal of the $40 \times 40 \mathrm{~mm}^{2}$ sensitive area of the detector. The total charge collected by the silicon detector is integrated and digitized by 12 bits ADC.

Figure 3 shows the measured amplitude spectrum on one detector for $45 \mathrm{GeV}$ (a) and $68 \mathrm{GeV}$ (b) beam energies. There is a peak for values above 100, mainly due to off-momentum particles hitting the detector. Their energy is about $1 \%$ lower than the real Bhabhas and the resolution of the calorimeter is not good enough to discriminate them. 
As we are only using one silicon detector, the fluctuations of the longitudinal development of the shower are large. Moreover, a fraction of the hits is close to the inner edge of the calorimeter, where part of the shower is not contained in the tungsten and remains undetected. This explains the broad amplitude spectra of Fig. 3.

By going from $45 \mathrm{GeV}$ to $68 \mathrm{GeV}$ the peak position is increased by the ratio of the energies, but the resolution* of the peaks does not change significantly.
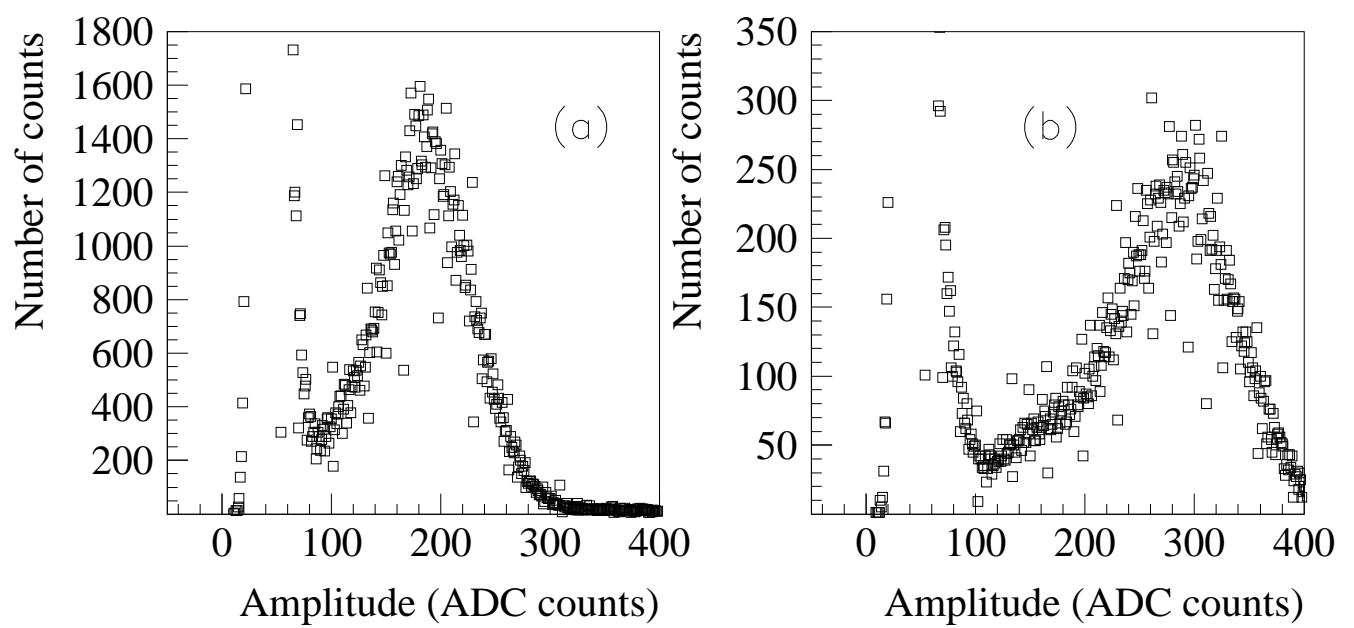

Figure. 3. Amplitude spectrum of $45 \mathrm{GeV}$ (a) and $68 \mathrm{GeV}$ (b) positrons measured in IP8. The threshold for high energy particle discrimination is at 100.

The peak observed for amplitudes lower than 100 is the noise due to the combination of the noise in the silicon detector and the noise in the $40 \mathrm{~m}$ cable between the detector and the electronics. In this example, the threshold for high energy particles tagging was set to 100.

\section{DATA ACQUISITION}

At each bunch crossing, the digitized signals from both internal and external calorimeters are read by a DSP(3). This DSP compares the signals of both calorimeters to the threshold values used to discriminate high energy particles. When the signals from both calorimeters are above their respective thresholds, a counter for pairs $N_{\text {pair }}$ is incremented by one. If only the signal from the internal detector is higher than the threshold, a counter for internals $N_{\text {int }}$ is incremented by one. The same rule applies to a counter for the externals $N_{\text {ext }}$. Finally, those three counters $N_{\text {pair }}, N_{\text {int }}$ and $N_{\text {ext }}$ are transferred to a microprocessor every $3 \mathrm{~s}$. Three of those basic acquisitions are performed before their average is sent to the control room.

*Defined as the ratio between the FWHM of the peak and its position. 


\section{LUMINOSITY}

The Bhabha rate and the background estimation are obtained from $N_{\text {pair }}$, $N_{\text {int }}$ and $N_{\text {ext }}$, thus allowing the complete determination of the luminosity and its statistical error.

\section{Background}

The luminosity detectors (cf Fig. 1) are protected on their back side (opposite to the interaction point) by the tungsten block of the collimator to which they are linked. This represents more than 30 radiation lengths, so no high energy particle can be detected from the back side.

However, from the front side, the detector can be hit by off-momentum particles. Even if a devoted set of collimators in the arcs and at the entrance of the straight section allows to reduce their rate, there are still some offmomentum particles hitting the detector, either coming from the arcs directly or from interaction with residual gas molecules or thermal photons in the straight section.

Synchrotron radiation photons have only several tens of keV. They do not induce any background, because their complete electromagnetic shower is absorbed in the first 5 radiation length of tungsten, and do not reach the silicon detector.

The only background which affects the detectors is then due to off-momentum particles. Table 1 shows the typical normalized background rates measured for the two different running energies of LEP in 1995 for each monitor. A typical current was about $300 \mu \mathrm{A}$ per bunch. The current normalized Bhabha rate is about $15 \mathrm{~Hz} / \mathrm{mA}$ at $45 \mathrm{GeV}$, which is two orders of magnitude smaller than the external background rate.

\begin{tabular}{|c|c|c|}
\hline & $\begin{array}{c}\text { Internal calorimeter } \\
(\mathrm{Hz} / \mathrm{mA})\end{array}$ & $\begin{array}{c}\text { External calorimeter } \\
(\mathrm{Hz} / \mathrm{mA})\end{array}$ \\
\hline \hline $45 \mathrm{GeV}$ & 0 to 120 & 600 to 1800 \\
$68 \mathrm{GeV}$ & 0 to 220 & 1800 to 3600 \\
\hline
\end{tabular}

Table 1. Typical normalized background rates measured in 1995 in each monitor.

The background rate is much higher in the detectors located on the external side of the ring as on the internal one. 


\section{Luminosity Calculation}

The accidental coincidence of background particles hitting at the same bunch crossing both the internal and the external detectors affects the coincidence pair rate $\dot{N}_{\text {pair }}$ computed from the counter $N_{\text {pair }}$. The Bhabha scattering rate $\dot{N}_{b}$ is :

$$
\dot{N}_{b}=\dot{N}_{p a i r}-\dot{N}_{a c c}
$$

were $\dot{N}_{a c c}$ is the accidental pair rate. With a statistical method(4) assuming uncorrelated background, the accidental pair rate $\dot{N}_{a c c}$ is calculated from the internal and external rates $\dot{N}_{i n t}$ and $\dot{N}_{\text {ext }}$. Finally, one obtains for the Bhabha rate $\dot{N}_{b}$ and its statistical error $\sigma\left(\dot{N}_{b}\right)$ :

$$
\begin{gathered}
\dot{N}_{b}=\dot{N}_{\text {pair }}-\frac{\dot{N}_{\text {int }} \dot{N}_{e x t}}{k f_{\text {rev }}-\dot{N}_{\text {int }}-\dot{N}_{\text {ext }}-\dot{N}_{\text {pair }}} \\
\sigma\left(\dot{N}_{b}\right) \approx \sigma\left(\dot{N}_{\text {pair }}\right)
\end{gathered}
$$

were $\sigma\left(\dot{N}_{\text {pair }}\right)$ is the statistical error on $\dot{N}_{\text {pair }}, f_{\text {rev }}$ is the revolution frequency and $k$ the number of bunches per beam. The correction term to $\dot{N}_{\text {pair }}$ in Eq. 3 is of the order of $50 \%$ at $45 \mathrm{GeV}$.

It is worthwhile noting that the often used delayed coincidence method has a much higher error(4).

\section{Statistical errors}

The statistical error $\sigma$ is computed by Eq. 4. An estimate of the quality of the background substraction is given by the fluctuations of the luminosity. If one considers two consecutive luminosity measurements $\mathcal{L}_{i}$ and $\mathcal{L}_{i+1}$, the rms of the difference $\mathcal{L}_{i+1}-\mathcal{L}_{i}$ divided by its error $\sqrt{\sigma_{i+1}^{2}+\sigma_{i}^{2}}$ must be one when the luminosity variations are only due to statistical fluctuations. This difference is plotted in Fig 4 were the line is a gaussian fit to the data.

To ensure that the luminosity was not affected by other sources than statistical fluctuations, the data used for Fig. 4 are registered during stable datataking conditions in beam separation scans (see next Section). The acquisition time for each point is $9 \mathrm{~s}$, giving a statistical uncertainty on each measurement of about $5 \%$. The shape of the histogram is well described by a gaussian with an RMS of $1.016 \pm 0.04$. This demonstrates that the statistical errors are estimated correctly.

\section{BEAM SEPARATION SCANS}

The optimization of the luminosity at LEP is done by measuring the luminosity for different separation of the electron and the positron beams. This separation is controlled by vertical electrostatic separators located on both sides of each interaction point. 


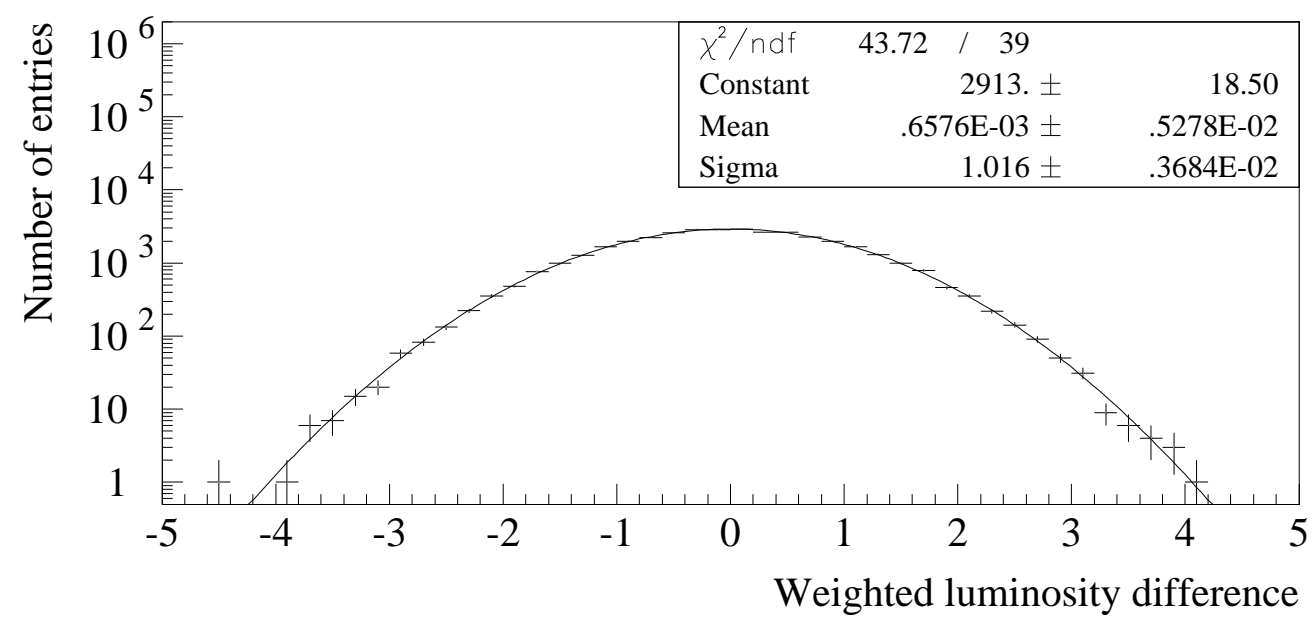

Figure. 4. Histogram of the difference between two consecutive luminosity measurements, divided by their combined error. The line is a gaussian fit to the data.

For each train crossing, the luminosity is recorded for the three families and plotted versus the beam separation. Figure 5 shows such a separation scan performed in one interaction point. The origin of the separation corresponds to the theoretical beam axis. Each point corresponds to an acquisition time of $27 \mathrm{~s}$. The curves are gaussian fits to the luminosity recorded for each family, the maximum of the gaussian being reached when the vertical separation between the electron and the positron bunches is zero for this family.

This method allows to find the optimum overlap between each family with a typical precision of $0.2 \mu \mathrm{m}$. The three families do not exactly overlap for the same value of the separator field, which is in agreement with simulations(5). The maximization of the luminosity is done by weighting the three individual optima.

The width $\sigma_{\text {scan }}$ of these individual gaussian curves is related to the vertical beam sizes $\sigma_{e^{+}}$and $\sigma_{e^{-}}$at the interaction point :

$$
\sigma_{\text {scan }}=\sqrt{\sigma_{e^{+}}^{2}+\sigma_{e^{-}}^{2}}
$$

For the example given in Fig. 5, assuming that both electron and positron beam sizes are equal, the measured vertical beam sizes are $4.9 \pm 0.3 \mu \mathrm{m}, 4.6 \pm 0.2 \mu \mathrm{m}$ and $3.7 \pm 0.2 \mu \mathrm{m}$ for family $\mathrm{A}, \mathrm{B}$ and $\mathrm{C}$ respectively.

This method is a unique way to measure the vertical beam sizes at the interaction point, with a typical precision of $0.2 \mu \mathrm{m}$.

\section{CONCLUSION}

The LEP luminosity monitors provide reliable on line luminosity measurements for all the colliding bunches. They are intensively used for luminosity 


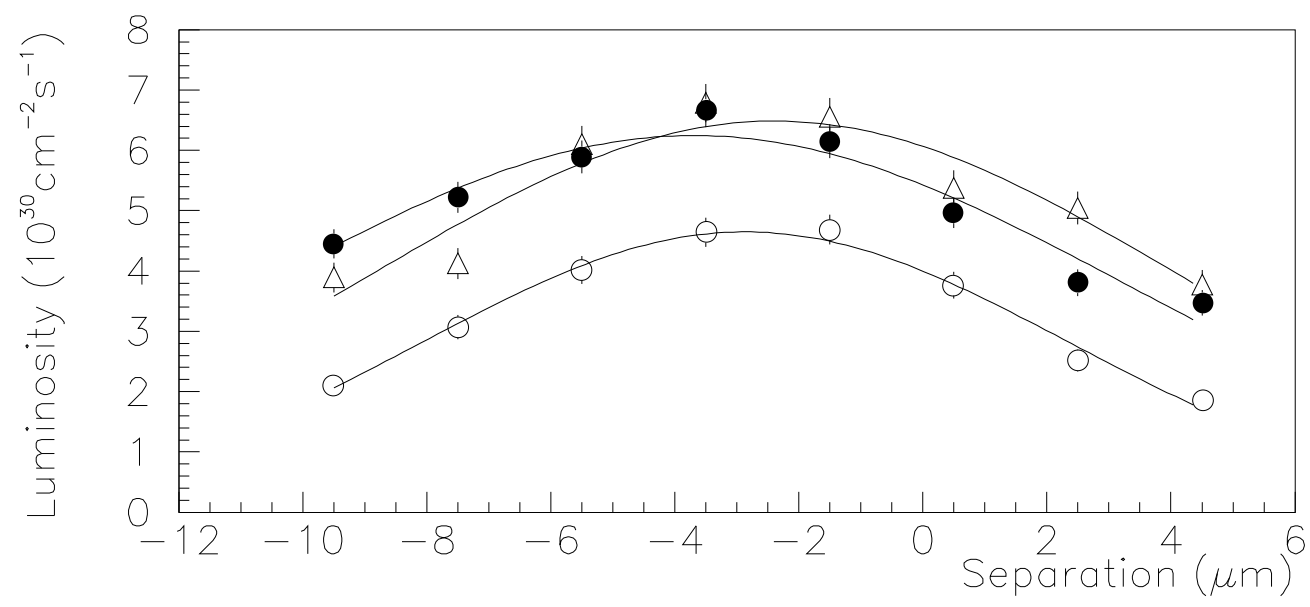

Figure. 5. Separation scan \#631 performed in IP2 for the three families : Family A $(\bullet)$, Family B $(\triangle)$ and Family $\mathrm{C}(\circ)$. The lines are gaussian fits to the data. The origin of the separation is the theoretical beam axis.

optimization and allow to measure the vertical beam sizes at the interaction point with very good accuracy.

\section{ACKNOWLEDGMENTS}

This work was performed in the CERN SL/BI group. The authors wish to thank M. Lamont and J. Wenninger for their implementation of the separation scan procedure.

\section{REFERENCES}

1. G. Altarelli, R. Kleiss and C. Verzegnassi, Z Physics at LEP 1, CERN Yellow report 89-08, Vol. 1 (1989)

2. G.P. Ferri, M. Glaser, G. von Holtey and F. Lemeilleur, Silicon Detectors Used for Beam Diagnostics in the LEP Collider, Nucl. Phys. B, Supplements Section (1990)

3. P. Castro, L. Knudsen, R. Schmidt, The Use of Digital Signal Processors in LEP Beam Instrumentation, Proceedings of the 3rd Annual Accelerator Instrumentation Workshop, CEBAF, Newport News, USA (October 1991), 253-259

4. P. Castro, Luminosity and beta function measurement at the electronpositron collider ring LEP, $\mathrm{PhD}$ thesis at the Valencia University, Spain (17th May 1996)

5. E. Keil, Truly self-consistent Treatment of the Side Effects with Bunch Trains, CERN SL/95-75 (1995) 